\title{
Animal-robot interaction and biohybrid organisms
}

\author{
Donato Romano ${ }^{1,2} \cdot$ Cesare Stefanini ${ }^{1,2,3}$
}

Published online: 18 November 2021

(c) The Author(s), under exclusive licence to Springer-Verlag GmbH Germany, part of Springer Nature 2021

This special issue on "Animal-Robot Interaction and Biohybrid Organisms" follows the pioneer "Workshop on AnimalRobot Interaction" organized by the guest editors at the International Conference on Intelligent Robots and Systems (IROS) 2020, aimed at consolidating the research community working on organism-robot systems.

Organisms and robots engaged in different ecological interactions represent biohybrid dynamic systems enabling new multidisciplinary scientific and technological investigations. In animals, evolutionary processes have led to the development of nervous systems allowing process information of biotic and abiotic events and effective reactions. Conversely, recent technology developments have produced relevant advances in robotics and bionics engineering, facilitating the design of artifacts exhibiting life-like appearance and behavior, and perception by animals as living conspecific or heterospecific agents. This in turn allows the inclusion of robots in animal populations and communities, and the emergence of animal-robot interactions.

This research field represents a paradigm shift in the study of animal behavior, with potential applications to the control of animal populations in agriculture, to the improvement of animal farming conditions, and to wildlife preservation. Indeed, animal-robot interactions can have a key role in improving animal wellness and environmental sustainability by mitigating the influence of human activities on ecosystems, thus contributing to emerging integrated approaches such as One Health and EcoHealth.

The use of interactive artificial agents to study animal intelligence relies on multiple disciplines, such as biomimet-

\section{Donato Romano}

donato.romano@santannapisa.it

1 The BioRobotics Institute, Sant'Anna School of Advanced Studies, Viale Rinaldo Piaggio 34, 56025 Pontedera, Pisa, Italy

2 Department of Excellence in Robotics and AI, Sant'Anna School of Advanced Studies, 56127 Pisa, Italy

3 Healthcare Engineering Innovation Center (HEIC), Khalifa University, Abu Dhabi, UAE ics, robotics, machine learning, biosystems engineering, and neuroethology. Animals are elective model organisms to develop new methods in those fields, thanks to their learning and memory capabilities, to their inter-individual differences, and to their adaptation to environmental perturbations. Furthermore, understanding animal intelligence through hybrid interactions with robots can be an effective strategy to solve challenges in real-world engineering applications and to enhance the capabilities of current bioinspired robotic systems, especially when robots have to interact with agents in natural environments, including humans.

Biohybrid systems can operate as distributed networks of sensors and actuators with animals and robots acting as advanced bio-artificial agents possessing unprecedented biohybrid cognitive and physical capabilities. In addition, co-locating living organisms and technological elements via symbiotic methods to form complex entities can allow the development of new types of biohybrid agents for multiple purposes, including environmental monitoring and management. The salient features of these biohybrid agents include energy harvesting (i.e., managed by microbial life forms that use organic matter as an energy source), low-power computing, biodegradability and biocompatibility, sensing, and actuation.

Articles in the Special Issue have proposed ethorobotics and animal-robot interaction as a novel translational neuroethological engineering approach with relevance to the study of cognition, and to real-world healthcare applications, as well as a new paradigm to face engineering and environmental challenges via sustainable approaches. Also, a philosophical view point has been provided by one author. The Guest Editors believe that this special issue of Biological Cybernetics, by combining biology and engineering, makes important scientific and technological contributions to a prolific key area for future robotics and bionics.

Finally and behalf of the editor-in-chief, we would like to mention that this special issue also includes two contributions unrelated to our topic: one by Hugh Wilson and Jack Cowan 
on their famous equation published in 1973 in this journal and another one by former Editor-in-Chief Leo van Hemmen on the role of core concepts in science in general and on core concepts put forward in Biological Cybernetics in particular. These two articles conclude the series of retrospective pieces celebrating the $60^{\text {th }}$ anniversary of this journal.

\section{Donato Romano and Cesare Stefanini.}

Publisher's Note Springer Nature remains neutral with regard to jurisdictional claims in published maps and institutional affiliations. 